\title{
Insuficiencia cardíaca con fracción de eyección preservada en un paciente con parálisis frénica bilateral. Cuando los biomarcadores no ayudan al diagnóstico
}

\author{
Heart failure with preserved ejection fraction in a patient with bilateral phrenic paralysis. \\ When the biomarkers do not help the diagnosis
}

\author{
Ramón Maseda-Uriza ${ }^{1 *}$, Alfonso Jurado-Román², Jesús Piqueras-Flores ${ }^{1}$, Raquel Frías-García1, \\ Pedro Pérez-Díaz y Juan A. Requena-Ibañez ${ }^{1}$
}

${ }^{1}$ Servicio de Cardiología, Sección de Cardiología Clínica; ${ }^{2}$ Servicio de Cardiología, Sección de hemodinámica. Hospital General Universitario de Ciudad Real, Ciudad Real, España

\section{Introducción}

La parálisis frénica bilateral es una rara causa de disnea, sobre todo en decúbito supino y al esfuerzo'. Muchas de estas parálisis están relacionadas con neuropatías y la neuralgia amiotrófica es una de ellas. Se trata de una rara entidad de etiología aún desconocida descrita por Parsonage y Turner en 1948; en muchos pacientes hay antecedentes de infecciones, intervenciones quirúrgicas o vacunas ${ }^{2}$. La insuficiencia cardíaca (IC) es la enfermedad cardíaca más frecuentemente diagnosticada, aproximadamente la mitad de los pacientes tienen una fracción de eyección preservada (ICFEp), cuyo síntoma principal es una intolerancia severa al ejercicio ${ }^{3}$. La coexistencia de otras comorbilidades como enfermedad pulmonar obstructiva crónica puede dificultar el diagnóstico de ICFEp.

\section{Caso clínico}

Presentamos a un varón de 45 años, de profesión pastor, no fumador, bebedor ocasional, que acude a urgencias por disnea de un mes de evolución con empeoramiento al ejercicio y al acostarse, que en la última semana presenta intolerancia al decúbito. Cuenta que desde hace un año presenta limitación al ejercicio y que el episodio actual comenzó tras un esfuerzo importante con dolor a nivel de ambos hombros, al cual no dio importancia. Al examen físico el paciente estaba taquipneico, con una saturación de oxígeno del 85\%, FC de 102 Ipm y TA de 180/95 mmHg; en la auscultación cardiopulmonar destacaba una disminución del murmullo vesicular en ambas bases pulmonares con crepitantes hasta campos medios. Inicialmente se diagnostica de IC «no filiada» y se inicia administración de oxígeno con mascarilla y $40 \mathrm{mg}$ de furosemida iv.

La radiografía de tórax (Figs. 1A y 1B) fue informada como mal inspirada, con signos de redistribución vascular y derrame izquierdo. Se solicita valoración por cardiología y se decide realizar determinación de niveles de BNP, que estaban elevados $(235 \mathrm{pg} / \mathrm{ml})$ y ecocardiograma transtorácico (ETT), en el que se evidencia una hipertrofia de ventrículo izquierdo (HVI)

\section{Correspondencia:}

*Ramón Maseda-Uriza

Servicio Cardiología

Hospital General Universitario Ciudad Real

Obispo Rafael Torija, s/n

Fecha de recepción: 02-06-2017

Fecha de aceptación: 10-09-2018

DOI: 10.24875/ACM.M19000002
Disponible en internet: 19-03-2019 Arch Cardiol Mex. 2019;89(1):78-81 www.archivoscardiologia.com 
concéntrica moderada, sin alteraciones de la contractilidad, con una fracción de expulsión del ventrículo izquierdo del $58 \%$. Los valores de la onda $E$ y $A$ fueron 0.6 y $0.2 \mathrm{~m} / \mathrm{s}$ respectivamente, una relación E/A de 3, que no se modificaba a las maniobras de Valsalva; el Doppler tisular a nivel de anillo mitral lateral mostró una onda $\mathrm{e}^{\prime}$ de $5 \mathrm{~cm} / \mathrm{s}$ (Figs. 2A y 2B) con una aurícula izquierda (Al) ligeramente dilatada $\left(38 \mathrm{ml} / \mathrm{m}^{2}\right)$, sin insuficiencia mitral, ventrículo derecho no dilatado con función conservada y una insuficiencia tricuspídea moderada que permitió calcular una presión sistólica de arteria pulmonar de $48 \mathrm{mmHg}$.

Con diagnóstico de ICFEp se decide ingreso a cargo de cardiología en tratamiento con diuréticos iv., inhibidores de la enzima convertidora de angiotensina y betabloqueantes. Al segundo día de ingreso y a pesar de adecuada diuresis, con balance hídrico negativo, no hay mejoría en la disnea ni la ortopnea, con intolerancia al decúbito y necesidad de dormir sentado por desaturación importante, precisando inicio de ventilación mecánica no invasiva (VMNI) en modo BiPAP.

Se realiza nueva radiografía de tórax sin cambios respecto a la previa y un nuevo ETT en el cual llama la atención un movimiento anómalo del septo interventricular hacia el ventrículo izquierdo en la sístole y una hipertensión pulmonar de $55 \mathrm{mmHg}$. En el examen físico se encontró una atrofia en la musculatura supraclavicular, deltoides con escapula alada (Figs. 1C y 1D) y respiración paradójica. Con la sospecha de otra causa de disnea se interconsulta a neumología, realizándose una espirometría que muestra una FEV1/FVC de 57.4, FEV1 de 0.83 । (22.6\%) y una FVC de 1.44 । (32.6\%) compatible con restricción severa; y a neurología, realizándose resonancia magnética cervical y dorsal, siendo un estudio muy limitado por la intolerancia al decúbito del paciente pero sin hallazgos relevantes; el estudio neurofisiológico informa de signos agudos de denervación en trapecios, supraespinosos y deltoides izquierdo; el estudio de conducción motora de nervio espinal accesorio derecho y axilar izquierdo muestra amplitud severamente reducida, el nervio espinal accesorio izquierdo con potencial motor moderadamente reducido, polineuropatía y plexitis. Con estos datos se hace el diagnóstico de neuralgia amiotrófica con afectación frénica bilateral. Se suspenden diuréticos y resto de medicación para IC y se inicia administración de corticoides, vitaminas del grupo B e inmunoglobulinas iv., manteniendo soporte ventilatorio con VMNI. A pesar del tratamiento persisten los signos de denervación con escasa recuperación funcional, precisando al alta soporte con VMNI.

\section{Discusión}

En este paciente la disnea claramente estaba relacionada con la parálisis frénica bilateral en el contexto de la neuralgia amiotrófica, sin embargo en el estudio ecocardiográfico hay datos que sugieren el diagnóstico de ICFEp (dilatación de Al, HVI y patrón de llenado restrictivo $^{3}$. La utilidad de biomarcadores para el diagnóstico diferencial de la disnea está demostrado y su elevación es uno de los criterios para el diagnóstico de ICFEp, en este caso la elevación del BNP fue determinante para asociar la disnea a una posible causa cardíaca, pues es uno de los criterios diagnósticos establecidos en las últimas guías de práctica clínica de la Sociedad Europea de Cardiología (ESC 2016), si además tenemos en cuenta que su rentabilidad aumenta en pacientes con incertidumbre en el diagnóstico ${ }^{4}$; en nuestro paciente había una cierta discrepancia entre la intensidad de la disnea y los hallazgos en las pruebas complementarias. El cuadro clínico clásico de la neuralgia amiotrófica consiste en antecedentes de dolor intenso en la musculatura escapular seguido de debilidad progresiva y atrofia de los músculos inervados por las raíces cervicales C5 y C6 ${ }^{2}$. La afectación frénica bilateral es muy rara en estos pacientes y hay muy pocos casos descritos en la literatura. La parálisis unilateral idiopática es más frecuente y común en hombres; entre las posibles causas que hay que descartar se encuentran los tumores de cuello y mediastino y los carcinomas broncogénicos ${ }^{5}$.La respiración paradójica en pacientes con parálisis frénica consiste en el movimiento hacia adentro de la pared abdominal durante la inspiración, este signo característico fue pasado por alto en la valoración inicial en Urgencias y durante los primeros días de estancia en la planta, dándose más importancia al síntoma (disnea). Es probable que en este caso, y dado los hallazgos del ecocardiograma, pudieran coexistir síntomas de ICFEp; además el paciente contaba clínica de intolerancia al ejercicio de un año de evolución. No consideramos que la elevación del BNP sea un falso positivo, lo que sí está claro es que la intensa disnea del último mes no era de causa cardíaca. Revisando los casos publicados de neuralgia amiotrófica con afectación frénica bilateral, la mayoría de ellos ha ingresado antes en neumología y son diagnosticados de alteraciones restrictivas por los resultados de las pruebas funcionales. Es característico en estos pacientes un empeoramiento de la capacidad vital en el decúbito por el desplazamiento de las vísceras abdominales al tórax. Para el diagnóstico es útil el uso de la fluoroscopia, que muestra el movimiento paradójico del diafragma; en el estudio 


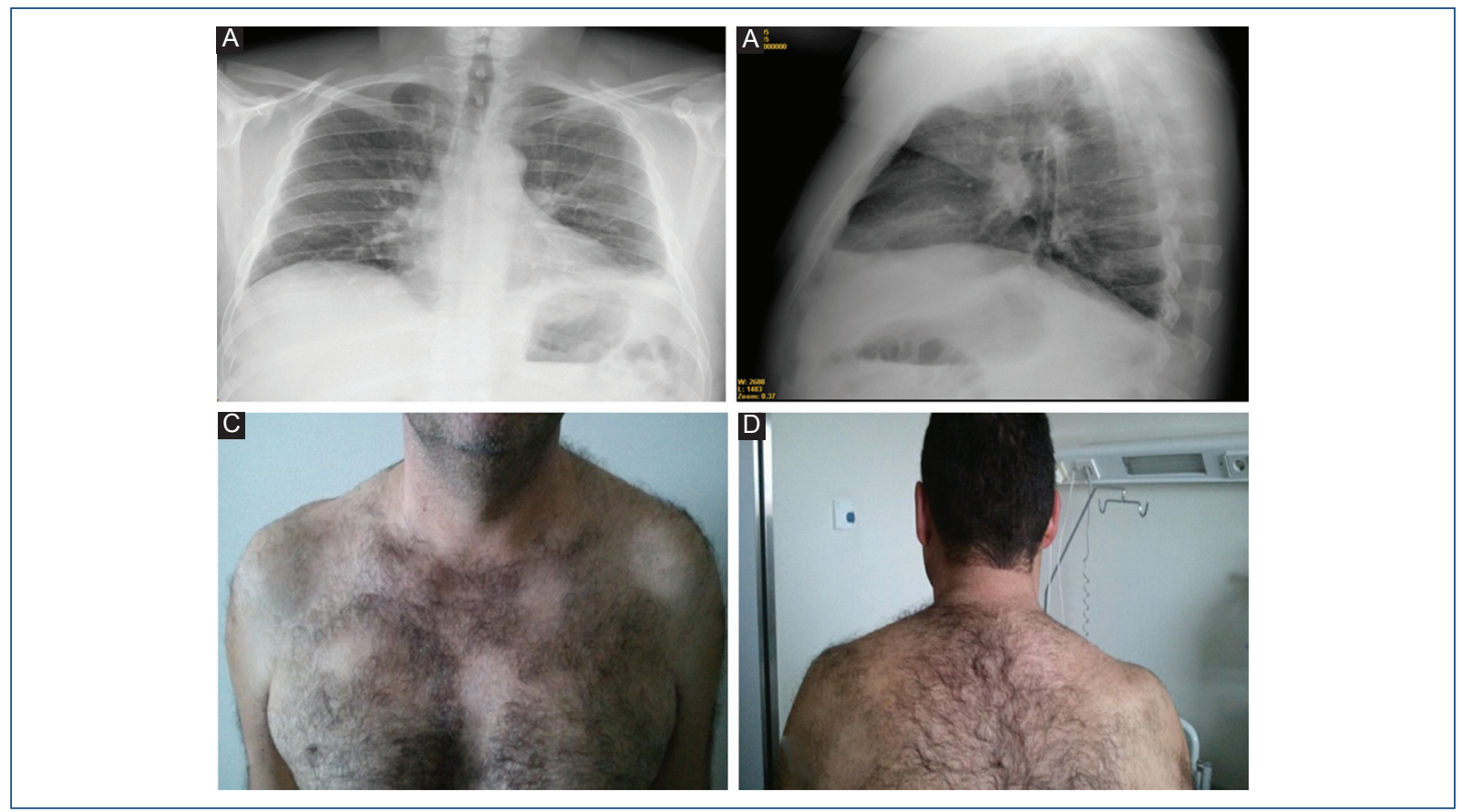

Figura 1. A y B: radiografía PA y lateral de tórax donde se observa elevación diafragmática. C y D: se puede observar la atrofia de la musculatura de cintura escapular, más evidente en lado derecho.

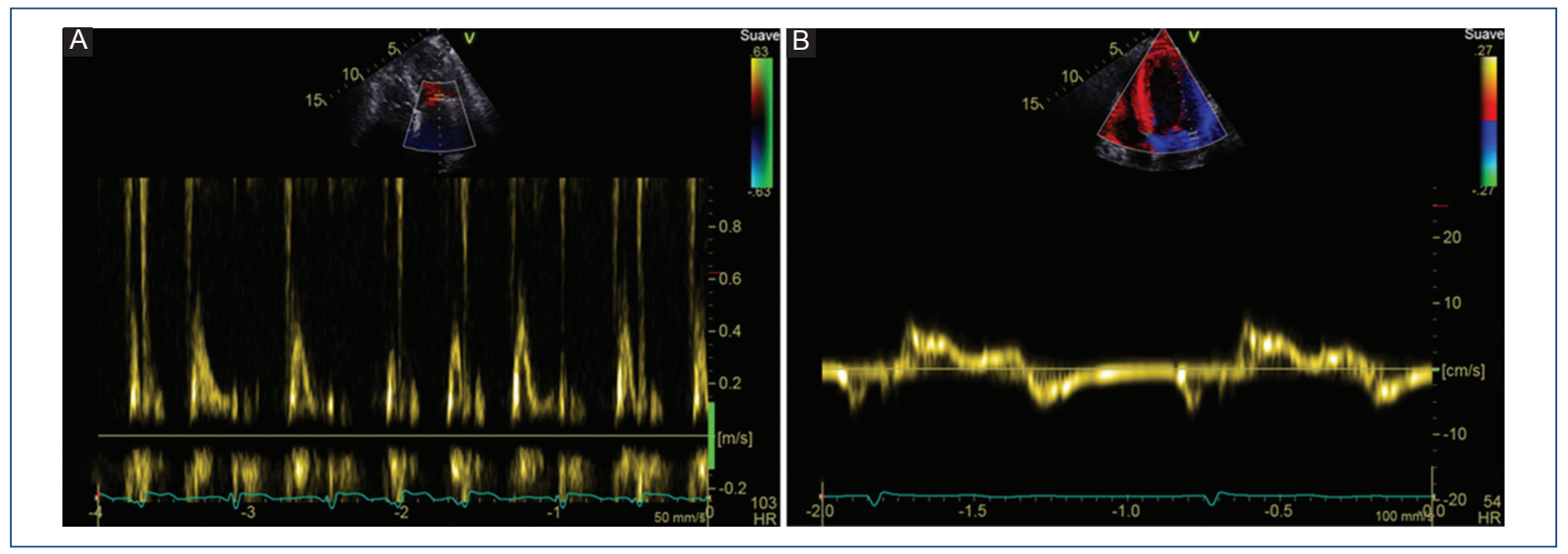

Figura 2. A: patrón de llenado mitral restrictivo. B: DTI anillo mitral lateral.

neurofisiológico es característico el enlentecimiento de las velocidades de conducción ${ }^{5}$. Para el tratamiento de la neuralgia amiotrófica se han empleado los corticoides o las inmunoglobulinas iv. con diversas tasas de éxito. En nuestro caso no hubo mejoría a pesar de emplear ambas opciones. Para el manejo de la parálisis frénica se utiliza la VMNI en modo BiPAP o CPAP; si no hay mejoría hay opciones quirúrgicas, como la plicatura diafragmática en el caso de parálisis unilateral o el implante de un marcapasos frénico ${ }^{6}$.

\section{Conclusiones}

A pesar de que en la medicina actual el uso de biomarcadores se impone para ayudar al diagnóstico diferencial de la disnea, es importante no olvidar que un buen examen físico puede orientarlo y no debe ser sustituido. La ICFEp puede coexistir con otras patologías que enmascaran su diagnóstico y por eso es importante tenerla en cuenta en el diagnóstico diferencial de la disnea. 


\section{Conflicto de intereses}

Los autores declaran no tener conflicto de intereses ni haber recibido financiación.

\section{Responsabilidades éticas}

\section{Protección de personas y animales}

Los autores declaran que para esta investigación no se han realizado experimentos en seres humanos ni en animales.

\section{Confidencialidad de los datos}

Los autores declaran que han seguido los protocolos de su centro de trabajo sobre la publicación de datos de pacientes.

\section{Derecho a la privacidad y consentimiento informado}

Los autores declaran que en este artículo no aparecen datos de pacientes.

\section{Bibliografía}

1. Ikegami G, Abe T, Akasaka K, Kouyama A, Souma R, Matsuo T, et al. Bilateral phrenic nerve paralysis manifested by orthopnea for 6 months in a patient with neuralgic amyotrophy. Intern Med. 2009;48(24):2123-7.

2. Lahrmann H, Grisold W, Authier FJ, Zifko UA. Neuralgic amyotrophy with phrenic nerve involvement. Muscle Nerve. 1999;22(4):437-42.

3. Montero D, Flammer AJ. Exercise intolerance in heart failure with preserved ejection fraction : time to scrutinize diuretic therapy ? Eur J Heart Fail. 2017;19(8):971-3.

4. Steinhart B, Thorpe KE, Bayoumi AM, Moe G, Januzzi JL Jr, Mazer CD. Improving the diagnosis of acute heart failure using a validated prediction model. J Am Coll Cardiol. 200913;54(16):1515-21.

5. Gayan-Ramirez G, Gosselin N, Troosters T, Bruyninckx F, Gosselink R, Decramer M. Functional recovery of diaphragm paralysis: a long-term follow-up study. Respir Med. 2008;102:690-8.

6. Rafiq A, ljaz M, Tariq H, Vakde T, Duncalf R. Failing phrenics: an obscure cause of exertional dyspnea: Case report and literature review. Medicine (Baltimore). 2016;95(29):42-63. 